\title{
INHALTSVERZEICHNIS BAND II
}

Vorwort ............................ V

Inhaltsverzeichnis Band I Einteilchenbewegung . . . . . . . . . . . . . . . . . XI

Kapitel 4 Rotationsspektren ... . . . . . . . . . . . . . . . . . . . 1

4-1 Auftreten von kollektiver Rotationsbewegung in Quantensystemen . . . . . 1

4-2 Symmetrien der Deformation. Rotationsfreiheitsgrade . . . . . . . . . . 3

4-2a Freiheitsgrade bei räumlichen Drehungen . . . . . . . . . . . . . . . . 4

4-2 b Folgerungen aus der Axialsymmetrie . . . . . . . . . . . . . . . . . . . . . . 5

4-2c $\mathscr{R}$-Invarianz . . . . . . . . . . . . . . . . . . . 6

4-2d $\quad \mathscr{P}$ - und $\mathscr{T}$-Symmetrie $\ldots \ldots \ldots \ldots$

4-2e Deformationen, die die $\mathscr{P}$ - oder $\mathscr{T}$-Symmetrie verletzen . . . . . . . . . 11

4-2f Kombinationen von Rotations- und Spiegelungssymmetrien . . . . . . . . 12

4-2g Rotation im Isospinraum . . . . . . . . . . . . . . . 16

4-3 Energiespektren und Intensitätsbeziehungen für axialsymmetrische Kerne $\quad 17$

4-3a Rotationsenergien . . . . . . . . . . . . . . . . . . 18

4-3 b E2-Matrixelemente innerhalb einer Bande . . . . . . . . . . . . . . . 35

4-3c $\quad M$ 1-Matrixelemente innerhalb einer Bande . . . . . . . . . . . . . . . 43

4-3d Allgemeine Struktur von Matrixelementen . . . . . . . . . . . . . . . 47

Beispiele zu Abschnitt 4-3 . . . . . . . . . . . . . . 50

4-4 Kopplung zwischen Rotation und innerer Bewegung bei axialsymmetrischen

Kernen . . . . . . . . . . . . . . . . . . . . . . . . . . . . 124

Beispiele zu Abschnitt 4-4 . . . . . . . . . . . . . . . . . . . . . . . 131

4-5 Rotationsspektren für Systeme ohne Axialsymmetrie . . . . . . . . . . . . 150

4-5a $\quad$ Symmetrieklassifizierung für gerade $A$. . . . . . . . . . . . . . . . . . 151

4-5b Energiespektren . . . . . . . . . . . . . . . . . . . . 155

4-5 c Systeme mit kleiner Asymmetrie . . . . . . . . . . . . . . . . . . . . . . . . . 159

4-5d $\quad$ Symmetrieklassifizierung für ungerade $A$. . . . . . . . . . . . . . . . . . . . . . 161

4-5e Zustände mit großem I . . . . . . . . . . . . . . . . . . . . . . . . . . . . . . . . . . 163

Beispiele zu Abschnitt 4-5 . . . . . . . . . . . . . . . . . . . . . . . . . . . . . 166

Anhang 4 A Teilchen-Rotor-Modell . . . . . . . . . . . . . . . . . 171

4A-1 Gekoppeltes System . . . . . . . . . . . . . . . . . . 171

4A-2 Adiabatische Näherung . . . . . . . . . . . . . . . . . . . . . . . . . . . . . . . 172

4A-3 Nichtadiabatische Effekte . . . . . . . . . . . . . . . . . . . . 175

Kapitel 5 Einteilchenbewegung in nichtsphärischen Kernen . . . . . 183

5-1 Stationäre Zustände der Teilchenbewegung in einem sphäroidalen Potential . 184

5-1 a Symmetrie und Form der Gleichgewichtsdeformation des Kerns . . . . . . 184 
5-1 b Deformiertes Potential . . . . . . . . . . . . . . . . 184

5-1 c Struktur der Einteilchenwellenfunktionen . . . . . . . . . . . . . . 186

Beispiele zu Abschnitt 5-1 . . . . . . . . . . . . . . . . . . . . . . . 189

5-2 Klassifizierung der Spektren von Kernen mit ungerader Massenzahl . . . . 208

5-3 Momente und thergänge . . . . . . . . . . . . . . . . . . 211

5-3a Einteilchentransfer . . . . . . . . . . . . . . . . . . . . 211

5-3b Einteilchenmomente und -übergänge . . . . . . . . . . . . . . . . . . 213

5-3c Paartransfer und $\alpha$-Zerfall . . . . . . . . . . . . . . . . . . . . . 215

5-3d Kopplung von Teilchen an die Rotationsbewegung . . . . . . . . . . . . 216

Beispiele zu Abschnitt 5-3 . . . . . . . . . . . . . . . . . . . . . . . 220

Anhang 5 A Streuung an nichtsphärischen Systemen . . . . . . . . . . 275

5A-1 Behandlung durch gekoppelte Kanäle . . . . . . . . . . . . . . 275

5 A-2 Adiabatische Näherung . . . . . . . . . . . . . . . . . . 278

Kapitel 6 Vibrationsspektren .................... 281

6-1 Einleitung . . . . . . . . . . . . . . . . . . . . . . . . . . . 281

6-2 Quantentheorie harmonischer Schwingungen . . . . . . . . . . . . 284

6-2a Erzeugungsoperatoren für Anregungsquanten . . . . . . . . . . . . . . 284

6-2 b Schwingungsamplituden .................. 285

6-2c Kollektivbewegung infolge eines schwingenden Einteilchenpotentials . . . . 287

6-3 Normalschwingungen des Kerns . . . . . . . . . . . . . . . . . . . . 293

6-3a Formschwingungen. Sphärische Gleichgewichtsform . . . . . . . . . . . 294

6-3b Schwingungen um einen sphäroidalen Gleichgewichtszustand . . . . . . . . 309

6-3c Kollektivbewegung beim Spaltproze $\ldots$. . . . . . . . . . . . . . . . . 313

6-3d Isospin von Schwingungen. Polarisations- und Ladungsaustauschschwingungen 321

6-3e Kollektive Schwingungen mit Spinfreiheitsgraden. . . . . . . . . . . . . 328

6-3f Zweinukleonentransfer. Paarschwingungen . . . . . . . . . . . . . . . 330

6-4 Summenregeln für Multipol-Oszillatorstärken . . . . . . . . . . . . . . 341

6-4 a Klassische Oszillatorsummen . . . . . . . . . . . . . . . . . . . . . 341

6-4 b Vibrationsoszillatorstärke in Einheiten der Summenregel . . . . . . . . . 346

6-4c Tensorsummen . . . . . . . . . . . . . . . . . . . . . . . . . 349

6-4d Ladungsaustauschbeiträge zur $E \lambda$-Oszillatorsumme . . . . . . . . . . . . . 352

6-5 Teilchen-Vibrationskopplung . . . . . . . . . . . . . . . . 355

6-5a Kopplungsmatrixelemente . . . . . . . . . . . . . . . . . . . . . . 356

6-5b Effektive Momente . . . . . . . . . . . . . . . . . . . . . . . . . 359

6-5c Matrixelemente für Einteilchentransfer . . . . . . . . . . . . . . . . . 362

6-5d Teilchen-Phonon-Wechselwirkungsenergie . . . . . . . . . . . . . . . . 363

6-5e Selbstenergien . . . . . . . . . . . . . . . . . . . . 366

6-5f Polarisationsbeiträge zu effektiven Zweiteilchenwechselwirkungen . . . . . 368

6-5g Effekte höherer Ordnung . . . . . . . . . . . . . . . . . . . . 369

6-5h Durch Teilchen-Vibrationskopplung angeregte Normalschwingungen . . . . 371

6-6 Anharmonische Effekte bei Vibrationsbewegung. Kopplung verschiedener

Anregungen . . . . . . . . . . . . . . . . . . . . . . . . . 381

6-6a Anharmonische Effekte bei niederfrequenten Quadrupolschwingungen . . . 382

6-6 b Kopplung von Quadrupol- und Dipolschwingungen . . . . . . . . . . . . 386

6-6c Rotations-Vibrationskopplung . . . . . . . . . . . . . . . . . . . 392

Beispiele zu Kapitel 6. . . . . . . . . . . . . . . . . . . . . . . . . . . 395

Responsefunktion . . . . . . . . . . . . . . . . . . . . . . . . 395

Eigenschaften von Dipolschwingungen $\left(\lambda \pi=1^{-}\right)$. . . . . . . . . . . . . 404

Eigenschaften von Quadrupolanregungen in sphärischen Kernen . . . . . . 434

Eigenschaften von Quadrupolschwingungen in deformierten Kernen. . . . . 472

Eigenschaften der Oktupolanregungen . . . . . . . . . . . . . . . . . 479

Sehalenstruktur in Einteilchenspektren . . . . . . . . . . . . . . . . . 499 
Schalenstruktureffekte in der Energie des Kerns . . . . . . . . . . . . . 517

Eigenschaften der Kernspaltung . . . . . . . . . . . . . . . . . . . . 532

Eigenschaften von Spinanregungen . . . . . . . . . . . . . . . . . 551

Eigenschaften der Paarkorrelationen . . . . . . . . . . . . . . . 556

Anhang 6A Tröpfchenmodell für Vibrationen und Rotationen . . . . . . 569

6A-1 Oberflächenschwingungen um die sphärisch symmetrische Gleichgewichtsform 569

6 A-2 Deformationen mit großer Amplitude. Spaltung . . . . . . . . . . . . . 576

6A-3 Kompressionsschwingungen . . . . . . . . . . . . . . . . . . . . . . 582

6A-4 Polarisationsschwingungen im Zwei-Flüssigkeiten-System . . . . . . . . . 585

6 A-5 Rotationsbewegung einer wirbelfreien Flüssigkeit . . . . . . . . . . . . 589

Anhang 6B Fünfdimensionaler Quadrupoloszillator . . . . . . . . . 591

6B-1 Form- und Winkelkoordinaten. Vibrations- und Rotationsfreiheitsgrade . . . 591

6B-2 Schwingungen um eine sphärische Gleichgewichtsform. . . . . . . . . . . 596

6B-3 Yrast-Bereich für harmonische Schwingungen . . . . . . . . . . . . . . 597

6B-4 Vielphononenzustände . . . . . . . . . . . . . . . . . . . . . . . . 601

Literatur (Band I und II) . . . . . . . . . . . . . . . . . . . . . . . . . . . . . 607

Sachverzeichnis (Band I und II) . . . . . . . . . . . . . . . . . . . . . . . . . . 636

\section{Inhaltsverzeichnis Band I Einteilchenbewegung}

Vorwort . . . . . . . . . . . . . . . . . . . . V

Kapitel 1 Symmetrien und Erhaltungssätze . . . . . . . . . . . . . . . . . . 1

1-1 Kernaufbau . . . . . . . . . . . . . . . . . . . . . . . 1

1-2 Beispiele zu Abschnitt 1-1 . . . . . . . . . . . . . . . . . . . . . 3

1-2a Kontinuierliche Transformationen

1-2b Raumspiegelung . . . . . . . . . . . . . . . . . . . . . 12

1-2c Zeitumkehr ....................... 15

1-3 Beispiele zu Abschnitt 1-2 . . . . . . . . . . . . . . . . . . . . . . . 20

1-3 Isobare Invarianz . . . . . . . . . . . . . . . . . . . . . . . . . 31

1-3a Isospinsymmetrie . . . . . . . . . . . . . . . . . . . . . . 31

1-3b Erweiterung der isobaren Symmetrie . . . . . . . . . . . . . . . . . . 37

Beispiele zu Abschnitt 1-3 . . . . . . . . . . . . . . . . . . . . . . . 41

1-4 Invarianzbedingungen für Kernkräfte . . . . . . . . . . . . . . . . . . 66

1-4a Geschwindigkeitsunabhängige Kräfte . . . . . . . . . . . . . 66

1-4b Geschwindigkeitsabhängige Kräfte . . . . . . . . . . . . . . . . . . . 68

Anhang 1A Drehinvarianz ........................ 70

1A-1 Drehimpulsmatrizen . . . . . . . . . . . . . . . . . 70

1 A-2 Kopplung von Drehimpulsen . . . . . . . . . . . . . . . . . . . . . . 71

1A-3 Umkopplungskoeffizienten . . . . . . . . . . . . . . . . . . . . . 73

1A-4 Drehmatrizen. D-Funktionen . . . . . . . . . . . . . . . . . . . . . 76

1A-5 Sphärische Tensoren und reduzierte Matrixelemente . . . . . . . . . . . 81

1A-6 Transformation in das innere Koordinatensystem . . . . . . . . . . . . . . . 89

1A-7 Transformation von Feldern . . . . . . . . . . . . . . . . . . . . 92

1A-8 Kopplung von Feldern und Entwicklung nach Multipolmomenten . . . . . . 94

1 A-9 Tensoren im Isospinraum . . . . . . . . . . . . . . . . . . 98 
Anhang 1 B Zeitumkehr . . . . . . . . . . . . . . . . . . . . . . . . . . . . . 99

1 B-1 Einteilchenzustände . . . . . . . . . . . . . . . . . . . . . . . . . . 99

1B-2 Vielteilchenzustände (gebundene Systeme) . . . . . . . . . . . . . . . . 101

1B-3 Stoßprozesse . . . . . . . . . . . . . . . . . . . . . 103

1B-4 Zerfallsprozesse . . . . . . . . . . . . . . . . . 105

Anhang 1C Permutationssymmetrie . . . . . . . . . . . . . . . . . 108

1C-1 Symmetriequantenzahlen (Partitionen) . . . . . . . . . . . . . . . . . 109

1 -2 Symmetrieklassifizierung von Wellenfunktionen im Raum der Besetzungszahlen 122

1C-3 Unitäre Symmetrie . . . . . . . . . . . . . . . . . . . . . . 127

Beispiele zu Anhang 1C . . . . . . . . . . . . . . . . . . . . . 134

Kapitel 2 Bewegung unabhängiger Teilchen . . . . . . . . . . . . . . . . . 145

2-1 Allgemeine Eigenschaften der Atomkerne . . . . . . . . . . . . . . . . 145

2-1 a Größe des Kerns . . . . . . . . . . . . . . . . . . . . . . . . 145

2-1 b Mittlere freie Weglänge der Nukleonen . . . . . . . . . . . . . . . . . 146

2-1 c Impulsverteilung (FrRMI-Gas-Näherung) . . . . . . . . . . . . . . . . . 147

2-1 d Bindungsenergien der Kerne . . . . . . . . . . . . . . . . . . . . . 148

2-1 e Paarungsenergie . . . . . . . . . . . . . . . . . . . . 150

2-1f Isospinquantenzahl . . . . . . . . . . . . . . . . . . . . . . . . . . 151

2-1g Kernpotential . . . . . . . . . . . . . . . . . . . . . . 154

2-1 h Antisymmetrische Wellenfunktionen des FerMi-Gases . . . . . . . . . . . 157

2-1 i Statistische Eigenschaften des Anregungsspektrums . . . . . . . . . . . . 160

Beispiele zu Abschnitt 2-1 . . . . . . . . . . . . . . . . . . . . . . . . 166

2-2 Schalenstruktur des Kerns . . . . . . . . . . . . . . . . . . . . . . . . . . . . 199

2-2a Bindungsenergien . . . . . . . . . . . . . . . . . . . . . . . . . . . 199

2-2b Anregungsenergien von gerade-gerade Kernen . . . . . . . . . . . . . . 200

2-2c Niveaudichten . . . . . . . . . . . . . . . . . . . . . 200

Beispiele zu Abschnitt 2-2 . . . . . . . . . . . . . . . . . . . . . . . . . 201

2-3 Kernarten und Häufigkeiten . . . . . . . . . . . . . . . . . . . . . . . 208

2-3a Stabilität der Kerne . . . . . . . . . . . . . . . . . . . . . . . . . . 208

2-3 b Relative Häufigkeiten und die Entstehung der Kernarten . . . . . . . . . 209

Beispiele zu Abschnitt 2-3 . . . . . . . . . . . . . . . . . . . . . . . 213

2-4 Mittleres Kernpotential . . . . . . . . . . . . . . . . . . . . . . . . 219

2-4a Reihenfolge der Einteilchenniveaus. Spinbahnkopplung . . . . . . . . . . 219

2-4 b Einteilchen-Stärkefunktion . . . . . . . . . . . . . . . . . 222

2-4c Optisches Potential . . . . . . . . . . . . . . . . . . . . . . . . . . 224

Beispiele zu Abschnitt 2-4 . . . . . . . . . . . . . . . . . . . . . . . . 231

2-5 Nukleonenwechselwirkungen und Kernpotential . . . . . . . . . . . . . . 253

2-5a Hauptmerkmale der Nukleonenwechselwirkung . . . . . . . . . . . . . . . 253

2-5b Beziehung zwischen Kernpotential und Nukleonenwechselwirkungen . . . . 264

2-5c Theorie der Kernmaterie . . . . . . . . . . . . . . . . . 275

Beispiele zu Abschnitt 2-5 . . . . . . . . . . . . . . . . . . . . . . . 276

Anhang 2A Antisymmetrisierte Produktfunktionen. Erzeugungs- und Vernichtungsoperatoren . . . . . . . . . . . . . . . . . . . . . . . 286

2A-1 Antisymmetrische Wellenfunktionen . . . . . . . . . . . . . . . . . . 286

2A-2 Eigenschaften der Erzeugungsoperatoren für Fermionen . . . . . . . . . . . . 287

2A-3 Einteilchenoperatoren . . . . . . . . . . . . . . . . . . 290

2A-4 Zweiteilchenoperatoren . . . . . . . . . . . . . . . . . . . . . . 291

2A-5 Teilchentransferoperatoren . . . . . . . . . . . . . . . . . . . . . . . 292

2A-6 $\quad x$-Darstellung . . . . . . . . . . . . . . . . . . . . . . . . . . . . 292

2A-7 Dichtematrizen . . . . . . . . . . . . . . . . . . . . . . . . . . . . 293

2A-8 Erzeugungsoperatoren für Bosonen . . . . . . . . . . . . . . . . . . . 294 
Anhang 2B Statistische Berechnung von Niveaudichten . . . . . . . . . . . . . . 296

2B-1 Niveaudichtefunktion und ihre LAPLACE-Transformierte . . . . . . . . . 296

2B-2 Inversion der LAPLACE-Transformation . . . . . . . . . . . . . . . . . 298

2B-3 Mittlere Besetzungszahlen für Einteilchenzustände . . . . . . . . . . . . . . 300

2B-4 Beschreibung des Spektrums durch Quasiteilchenanregungen . . . . . . . . . 301

2B-5 Thermodynamische Deutung der Niveaudichteberechnung . . . . . . . . . 303

2B-6 Berechnung von Niveaudichten, die durch zusätzliche Quantenzahlen bestimmt

werden . . . . . . . . . . . . . . . . . . . . . . 304

Anhang 2C Beschreibung von Schwankungen mit Hilfe stochastischer Matrizen . . . . . 310

2C-1 Stochastische Verteilung der Elemente einer zweidimensionalen Matrix . . . 310

2C-2 Verteilung der Eigenwerte und Eigenvektoren . . . . . . . . . . . 312

2C-3 Matrizen großer Dimension . . . . . . . . . . . . . . . . . . . 314

Anhang 2D Modell für Eigenschaften der Stärkefunktion . . . . . . . . . . . . . . 318

2D-1 Wahl der Darstellung . . . . . . . . . . . . . . . . . . . . . . . 318

2D-2 Diagonalisierung . . . . . . . . . . . . . . . . . . . . . 319

2D-3 Stärkefunktion für konstante Matrixelemente . . . . . . . . . . . . . . . 319

2D-4 Zeitabhängige Beschreibung des Kopplungsprozesses . . . . . . . . . . . 320

2D-5 Moment zweiter Ordnung der Stärkefunktion . . . . . . . . . . . . . . . . 321

2D-6 Zwischenstadien der Kopplung . . . . . . . . . . . . . . . . . . . . 321

2D-7 Berechnung der Stärkefunktion für nichtkonstante Matrixelemente . . . . . 322

Kapitel 3 Einteilchenkonfigurationen . . . . . . . . . . . . . . . . 325

3-1 Quantenzahlen und Wellenfunktionen. Teilchen-Loch-Symmetrie . . . . . 325

3-1 a Einteilchenzustände . . . . . . . . . . . . . . . . . . . . . 325

3-1 b Lochzustände. Teilchen-Loch-Konjugation . . . . . . . . . . . . . . . . 327

3-1 c Isospin für Teilchen- und Lochzustände . . . . . . . . . . . . . . . . . 329

Beispiele zu Abschnitt 3-1 . . . . . . . . . . . . . . . . . . . . . . . 330

3-2 Energiespektren . . . . . . . . . . . . . . . . . . . . . 332

Beispiele zu Abschnitt 3-2 . . . . . . . . . . . . . . . . . . . . . . . . 334

3-3 Matrixelemente elektromagnetischer Momente . . . . . . . . . . . . . . 348

3-3a Quadrupolmomente und E2-Utbergangswahrscheinlichkeiten . . . . . . . . 348

3-3b Magnetische Momente . . . . . . . . . . . . . . . . . . . . . 351

3-3c Andere elektromagnetische Momente . . . . . . . . . . . . . . . . . . 356

Beispiele zu Abschnitt 3-3 . . . . . . . . . . . . . . . . . . . . . . . . 356

3-4 Matrixelemente für $\beta$-Zerfall . . . . . . . . . . . . . . . . . . . . . . 360

3-4a Erlaubte Ubergänge . . . . . . . . . . . . . . . . . . 360

3-4b Verbotene Ubergänge . . . . . . . . . . . . . . . . . . . . . . 364

Beispiele zu Abschnitt 3-4 . . . . . . . . . . . . . . . . . . . . . . . 365

3-5 Reaktionen. Spektroskopische Amplituden . . . . . . . . . . . . . . . 370

3-5a Einteilchen-Transferreaktionen . . . . . . . . . . . . . . . 370

3-5b Resonanzreaktionen . . . . . . . . . . . . . . . . . . . . 371

Beispiele zu Abschnitt 3-5 . . . . . . . . . . . . . . . . . . . . . . . 372

Anhang 3A Einteilchenwellenfunktionen und -matrixelemente . . . . . . . . . . 376

3A-1 Kopplung von Spin und Bahnbewegung . . . . . . . . . . . . 376

3A-2 Berechnung der Matrixelemente von Einteilchenoperatoren . . . . . . . . . 379

Anhang 3B Teilchen-Loch-Konjugation . . . . . . . . . . . . . . . 384

3B-1 Beschreibung von Fermionensystemen durch Teilchen- und Lochzustände . . 384

3B-2 Matrixelemente von Einteilchenoperatoren . . . . . . . . . . . 388

3B-3 Matrixelemente von $\mathrm{Zweiteilchenoperatoren} \mathrm{.} \mathrm{.} \mathrm{.} \mathrm{.} \mathrm{.} \mathrm{.} \mathrm{.} \mathrm{.} \mathrm{.} \mathrm{.} \mathrm{.} 391$ 
Anhang 3C Matrixelemente für elektromagnetische Wechselwirkungen . . . . . . . . . 398

3C-1 Kopplung von Feld und Strom . . . . . . . . . . . . . . . . . . . . 398

3C-2 Strahlungsprozesse . . . . . . . . . . . . . . . . . . . . . . . . . . . . 399

3C-3 Wechselwirkungen mit geladenen Teilchen . . . . . . . . . . . . . . . 402

3C-4 Ladungs- und Stromdichte für freie Nukleonen . . . . . . . . . . . . . . 404

3C-5 Einteilchenmatrixelemente . . . . . . . . . . . . . . . . . 407

3C-6 Wechselwirkungseffekte im Strom . . . . . . . . . . . . . . . . 410

Anhang 3D Beta-Wechselwirkung . . . . . . . . . . . . . . . . 417

3D-1 Prozesse mit schwacher Wechselwirkung und schwacher Strom . . . . . . . 417

3D-2 Symmetrieeigenschaften des $\beta$-Stromes . . . . . . . . . . . . . 421

3D-3 Nichtrelativistische Form des $\beta$-Stromes . . . . . . . . . . . . . . . . 426

3D-4 Multipolmomente . . . . . . . . . . . . . . . . . . . . 429

3D-5 tt-Werte . . . . . . . . . . . . . . . . . . . . 434

Beispiele zu Anhang 3D . . . . . . . . . . . . . . . . . . . . 439

Anhang 3E Nukleon-Transferreaktionen . . . . . . . . . . . . . . . . . . . 445

3E-1 Einteilchen-Transferreaktionen . . . . . . . . . . . . . . 446

3E-2 Zweiteilchen-Transferreaktionen . . . . . . . . . . . . . . . . . . . .451

Anhang 3F Resonanzreaktionen . . . . . . . . . . . . . . . . . . . . . . . .454

3F-1 Allgemeine Merkmale der Resonanzstreuung . . . . . . . . . . . . . . . 454

3F-2 Resonanzparameter für die Einteilchenbewegung . . . . . . . . . . 465

Literatur . . . . . . . . . . . . . . . . . . . . . . 477

Sachverzeichnis . . . . . . . . . . . . . . . . . . . . . . . 491 